\begin{tabular}{c}
\hline \hline Juntendo \\
Research Profiles \\
\hline \hline Juntendo Medical Journal \\
2015. $61(3), 312-313$
\end{tabular}

\title{
Department of Gastroenterology
}

\section{Principal Investigator: Sumio Watanabe (Professor)}

The Department of Gastroenterology of Juntendo University was established in 1968. Today, about 100 physicians belong to this department, which is one of the largest clinical departments at Juntendo University. The target organs of our department may be thought to be gastrointestinal tract organs (esophagus, stomach, and colon). However, our department is committed to diagnosing and treating diseases not only in the esophagus, stomach, and colon, but also in a variety of organs, such as the small intestine, liver, gallbladder, pancreas, and peritoneum. About 65,000 outpatients attend our department annually, along with 100 to 120 inpatients at any time. We undertake substantial clinical and basic research for our patients regarding these organs and diseases. Our department is classified into four main research groups: upper gastrointestinal tract group, lower gastrointestinal tract group, liver disease group, and hepato-biliary-pancreatic group. Many physicians belong to each of these groups and undertake research on interesting themes. You are welcome to join our team and study with us.

\section{Group Leaders and Research Areas \\ 1) Mariko Hojo (Associate Professor), Daisuke Asaoka (Associate Professor), Kenshi Matsumoto (Associate Professor), Junko Kato (Associate Professor), Nobuko Serizawa (Associate Professor) Upper gastrointestinal tract}

We have investigated the pathophysiology of gastroesophageal reflux disease (GERD), histological markers for laryngo-pharyngeal reflux disease, Helicobacter pylori (H. pylori)-related gastric cancer involving epigenetic changes, and carcinogenesis-related SNPs of fundic gland gastric cancer, which is a gastric cancer without $H$. pylori infection, as basic research.

We have investigated the endoscopic and clinicopathological characteristics of fundic gland gastric cancer, ulcer healing after endoscopic mucosal dissection, the appropriate use of propofol sedatives during endoscopic mucosal dissection, the clinicopathological characteristics of gastric cancer after eradication, the relationship between mucosal dam- age and anticoagulant drugs, chronological changes in the rate of $H$. pylori eradication, and therapeutic effects of functional dyspepsia and GERD, as clinical research.

Publications:

1) Nagahara A, Hojo M, et al: A randomized prospective study comparing the efficacy of on-demand therapy versus continuous therapy for 6 months for long-term maintenance with omeprazole $20 \mathrm{mg}$ in patients with gastroesophageal reflux disease in Japan. Scand J Gastroenterol, 2014; 49: 409-417.

2) Ueyama H, Matsumoto K, et al: Gastric adenocarcinoma of the fundic gland type (chief cell predominant type). Endoscopy, 2014; 46: 153-157.

3) Matsumoto K, Nagahara A, et al: Development and clinical usability of a new traction device "medical ring" for endoscopic submucosal dissection of early gastric cancer. Surg Endosc, 2013; 27: 3444-3451.

\section{Chemotherapy}

Our group has been working on gastrointestinal cancer chemotherapy from various perspectives. We also contribute to some international multi-center clinical trials as well as domestic clinical trials. Here, we show our ongoing clinical and basic research.

Clinical research: 1) Clinical trial to evaluate the safety and efficacy of peptide vaccination for cancer patients. 2) Management tips for decreasing the adverse events of molecularly-targeted drugs for metastatic colorectal cancer. 3) Evaluation of the QOL in cancer patients undergoing chemotherapy. 4) Clinical trial for the development of a new effective chemotherapy regime. Basic research: 1) Galectin-3 regulates gastric cancer cell proliferation through a signaling pathway. 2) Association of serum DAO activity and gastrointestinal toxicity in advanced gastric cancer patients receiving chemotherapy. Publications:

1) Higashihara Y, Kato J, et al: Phase I clinical trial of peptide vaccination with URLC10 and VEGFR1 epitope peptides in patients with advanced gastric cancer. Int J Oncol, 2014; 44: 662-668.

2) Kato J, Nagahara A, et al: Phase I study of paclitaxel, cisplatin and 5-fluorouracil combination chemotherapy for unresectable/recurrent gastric cancer. Adv Med Sci, 2010; 55: 137-142.

3) Kato J, Nagahara A, et al: Evaluation of EORTC QLQC30 questionnaire in patients undergoing in-hospital chemotherapy for gastrointestinal cancer in Japan. J Gastroenterol Hepatol, 2008; 23 (Suppl 2) : S268-272. 
2) Taro Osada (Senior Associate Professor), Naoto Sakamoto (Associate Professor), Tomoyoshi Shibuya (Associate Professor)

Lower gastrointestinal tract

Our group has conducted basic and clinical research on the lower gastrointestinal tract as follows: [Basic research] 1) $\beta$-catenin expression and KRAS gene alteration in laterally spreading colorectal tumors. 2) HIF1 $\alpha$, PTCH, EphB2, or DNA-repair protein expression and BRAF mutation in colorectal serrated adenoma. 3) KRAS/BRAF mutation and mucin phenotypes in colorectal submucosal carcinoma. 4) An association of $\mathrm{WNT} / \beta$-catenin signal activation with methylation of genes in the serrated neoplasia pathway of the colorectum. 5) Mucin phenotypes in sessile serrated adenoma/polyp with dysplasia and carcinoma. 6) Micro-RNA expression in sessile serrated adenoma/polyp with dysplasia and carcinoma. 7) Methylation of DNA repair genes in ulcerative colitis-associated colorectal cancer. [Clinical research] 1) Histological distinction between the granular and nongranular types of laterally spreading tumors of the colorectum. 2) Traction device-assisted endoscopic submucosal dissection of large superficial colorectal tumors. 3) Clinicopathologic characteristics of sessile serrated adenoma/polyp with dysplasia and carcinoma.

Publications:

1) Murakami T, Mitomi H, et al: Distinct WNT/ $\beta$-catenin signaling activation in the serrated neoplasia pathway and the adenoma-carcinoma sequence of the colorectum. Mod Pathol, 2015; 28: 146-158.

2) Ritsuno H, Sakamoto N, et al: Prospective clinical trial of traction device-assisted endoscopic submucosal dissection of large superficial colorectal tumors using the S-O clip. Surg Endosc, 2014; 28: 3143-3149.

3) Osada T, Sakamoto N, et al: Process of wound healing of large mucosal defect areas that were sutured by using a loop clip-assisted closure technique after endoscopic submucosal dissection of a colorectal tumor. Gastrointest Endosc, 2013; 78: 793-798.

\section{Inflammatory bowel diseases and small intestine}

Our group is working on inflammatory bowel disease (IBD). We aim to elucidate the mechanism of its pathogenesis and pathophysiology and to establish novel treatment methods. Ongoing projects are as follows: 1) Effects of lymphocytes on the disease activity, especially focusing on mucosal-associated invariant T cells. 2) Functions of intestinal microflora and its potential for novel treatment. 3) Efficacy and functional mechanism of cytapheresis in IBD patients. 4) Predictive factors of relapse in ulcerative colitis patients. 5) Examination of small intestinal lesions using a capsule and double-balloon endoscopy in order to clarify their pathogenesis.

Publications:

1) Ishikawa D, Okazawa A, et al: Tregs are dysfunctional in vivo in a spontaneous murine model of Crohn's disease. Mucosal Immunol, 2013; 6: 267-275.

2) Shibuya T, Osada T, et al: Jejunal capillary hemangioma treated by using double-balloon endoscopy (with video). Gastrointest Endosc, 2010; 72: 660-661.

3) Osada T, Ohkusa T, et al: Comparison of several activity indices for the evaluation of endoscopic activity in UC: inter- and intraobserver consistency. Inflamm Bowel Dis, 2010; 16: 192-197.

3) Kenichi Ikejima (Associate professor), Satoko Suzuki (Associate professor), Shunhei Yamashina (Associate professor), Kazuyoshi Kon (Associate professor), Akira Uchiyama (Associate professor)

\section{Liver disease}

Our nationally and internationally recognized liver investigative group conducts a wide range of basic, epidemiologic, and clinical research. There are ongoing programs investigating the treatment of viral hepatitis, the molecular mechanisms of alcoholic liver injury, non-alcoholic fatty liver disease, and liver fibrogenesis. The following are particular liver diseases. Clinical research: 1) The relationship between liver diseases and lifestyle-related diseases. 2) The treatment and prognosis of chronic viral hepatitis. 3) The diagnoses of $\mathrm{PBC}$ and AIH. 4) The usefulness of endoscopic injection sclerotherapy and endoscopic variceal ligation for esophageal varices. Basic research: 5) Mitochondrial abnormalities and cellular autophagy in cancers and lifestyle-related diseases. 6) The role of lipid metabolism in liver disease. 7) Pathogenesis and mechanism of the progression of alcoholic liver disease. 8) The inhibition of activation of stellate cells and liver fibrogenesis. 9) The modulation of intestinal functions through the immune system and pharmacological nutrition support by amino acids.

Publications:

1) Fukuo Y, Yamashina S, et al: Abnormality of autophagic function and cathepsin expression in the liver from patients with non-alcoholic fatty liver disease. Hepatol Res, 2014; 44: 1026-1036.

2) Hosoya $\mathrm{S}$, Ikejima $\mathrm{K}$, et al: Innate immune responses involving natural killer and natural killer T cells promote liver regeneration after partial hepatectomy inmice. Am J Physiol Gastrointest Liver Physiol, 2013; 304: G293299.

3) Yamagata H, Ikejima K, et al: Altered expression and function of hepatic natural killer $\mathrm{T}$ cells in obese and diabetic KK-A (y) mice. Hepatol Res, 2013; 43: 276-288. 